\title{
Reflective scattering effects in double-pomeron exchange processes
}

\author{
S.M. Troshin, N.E. Tyurin \\ Institute for High Energy Physics, \\ Protvino, Moscow Region, 142281 Russia
}

\begin{abstract}
We discuss energy dependence of rapidity gap survival probability in the double-pomeron exchange processes with account of the reflective scattering effects.
\end{abstract}




\section{Introduction}

Double-pomeron exchange processes are the subject of the active theoretical studies since the beginning of the nineties, when the possibility to discover the Higgs production in these processes was discussed in the seminal papers [1, 2, 3]. Nowadays these studies are motivated by the current experiments at Tevatron [4] and planning for the future experimental studies at the LHC [5]. Needless to say that the processes with large rapidity gaps are important tool in the search of new physics. The extensive studies of the magnitude and energy dependence of gap survival probability have been performed, results of these studies together with the estimates of the Higgs production cross-section can be found, e.g. in papers [6, 7, 8, 9, 10, 11]. Recent results and review can be found in [12].

Perturbative QCD calculations can be used for calculations of the cross-sections of such processes where hard scale such as a large mass is present. But the necessity to take into account soft rescatterings in initial and final states leads to uncertainties related to the absence of the theoretically well established procedure of the multiparticle amplitude unitarization which is commonly reduced to the absorptive corrections due to the rescattering processes. Dynamics of such interactions in hard diffraction processes is accounted then by introduction of a factor which is known as a gap survival probability [3], i.e. a probability to keep away inelastic interactions which can result in filling up by hadrons the large rapidity gaps.

The gap survival probability (SP) was denoted as $\left\langle|S|^{2}\right\rangle$ since it was defined by the following relation [3]:

$$
\left\langle|S|^{2}\right\rangle=\frac{\int_{0}^{\infty} D_{H}(b)|S(s, b)|^{2} d^{2} b}{\int_{0}^{\infty} D_{H}(b) d^{2} b},
$$

where $D_{H}(b)$ is the probability to observe a specific hard interaction in collision of the hadrons $h_{1}$ and $h_{2}$ and $|S(s, b)|^{2}$, where $S$ is elastic scattering $S$-matrix, is a probability of the absence of the inelastic interactions. The above definition of SP proceeds from the eikonal scattering picture [13] which is usually used for the estimates of $\left\langle|S|^{2}\right\rangle$. It implies that the soft scattering picture at very high energy is an absorptive one $(S \geq 0)$ and the elastic scattering amplitude in the impact parameter representation does not exceed black disk limit which is a half of the unitarity limit. It is worth to note that the diffractive processes (with exclusion of elastic scattering) are inelastic processes themselves and therefore give contribution to absorption. Account for the soft processes is model dependent and strongly depends on the details of the particular model. Estimates for the gap survival probabilities can vary in the wide range, e.g. from 0.4 to $22 \%$ [14]. Of course, such wide variation of theoretical predictions is not encouraging for the experimental studies. 
Moreover, there are theoretical problems with the definition given by Eq. (1). As it was shown in [15], at very high energies the elastic scattering can go beyond the black disk limit [16] and become a reflective at small impact parameters, i.e. elastic $S$ matrix can have negative values. The generic geometrical picture at fixed energy beyond the black disc limit can be described in this case as a scattering off the partially reflective and partially absorptive disk surrounded by the black ring which becomes grey at larger values of the impact parameter. The evolution with energy is characterized by increasing albedo due to the interrelated increase of reflection and decrease of absorption at small impact parameters. This picture implies that the scattering amplitude at the LHC energies is beyond the black disk limit at small impact parameters (elastic $S$-matrix is negative) and it provides explanation for the regularities observed in cosmic rays studies [15]. The straightforward use of Eq. (1) [17] in this case leads to the non-monotonic energy dependence of SP and its increasing dependence. It approaches unity at very high energies. However, this limit is merely a manifestation of the pure reflective elastic scattering $(S \rightarrow-1)$ at $s \rightarrow \infty$ and $b=0$. The reflective elastic scattering does not result from absorption and therefore the definition given by Eq. (1) should be modified. The limitation of the definition Eq. (1) to absorptive scattering is absent if one uses the more general definition for the SP (we denote this quantity as $R_{s}^{2}$ to differentiate it from the Eq. (1)) given in [14, 18, 19]

$$
R_{s}^{2}(s)=\frac{\sigma_{d i f f}(\text { phys })}{\sigma_{d i f f}(\text { hard })},
$$

where $\sigma_{d i f f}($ hard $)$ can, in principle, be calculated with perturbative QCD, while $\sigma_{d i f f}$ (phys) is the real cross-section, which can be confronted with the crosssection measured experimentally. The definitions Eqs. (1) and (2) are equivalent if $S$ is nonnegative in the whole range of variation of the variables $s$ and $b$. However, they are not equivalent if $S$ can become negative at some values of the energy and impact parameter.

We use therefore the definition Eq. (2) and calculate the energy dependence of $R_{s}^{2}(s)$ in the unitarization scheme which includes reflective scattering in the double-pomeron exchange processes (DPE).

Now a few comments are to be added. First of all, of course, the quantity $R_{s}^{2}$ is model and process dependent. Another important point was mentioned in [9], this quantity is probability of surviving rapidity gaps, it is not the probability of production and surviving rapidity gaps. It is useful to have it in mind under discussion of the available experimental data on hard hadronic diffraction at Tevatron.

Experimental studies at Tevatron energies $\sqrt{s}=630 \mathrm{GeV}$ and $\sqrt{s}=1800$ $\mathrm{GeV}$ show that in the hard double-diffractive (DD) interactions the ratio of the of the corresponding event numbers decreases with energy [4]. The studies of inclusive DD at CDF [4] leads to conclusions on the universality of SP in soft 
and hard diffractive processes and on the absence of suppression of the additional gaps compare to the diffractive processes with single rapidity gap, i.e. SP does not depend on number of gaps.

\section{SP in double-pomeron exchange processes; effects of the reflective scattering}

As it was already mentioned, almost all estimates of SP are performed on the basis of the eikonal amplitude unitarization scheme. To strengthen the obtained predictions it is desirable to have predictions based on the other forms of the amplitude unitarization. It is important to move this way in the situation when there is no unique scheme for the unitarity account.

Alternative form of unitarization uses the rational representation for the scattering amplitude. The rational form of unitarization in quantum field theory is based on the relativistic generalization [20] of the Heitler equation [21]. In this $U$-matrix approach the elastic scattering matrix in the impact parameter representation has the form:

$$
S(s, b)=\frac{1+i U(s, b)}{1-i U(s, b)}
$$

where $S(s, b)=1+2 i f(s, b)$ and $U(s, b)$ is the generalized reaction matrix, which is considered to be an input dynamical quantity similar to the eikonal function. Unitarity equation rewritten at high energies for the elastic amplitude $f(s, b)$ has the form

$$
\operatorname{Im} f(s, b)=|f(s, b)|^{2}+\eta(s, b)
$$

where the inelastic overlap function

$$
\eta(s, b) \equiv \frac{1}{4 \pi} \frac{d \sigma_{\text {inel }}}{d b^{2}}
$$

is the sum of all inelastic channel contributions. Inelastic overlap function is related to $U(s, b)$ according to Eqs. (3) and (4) as follows

$$
\eta(s, b)=\frac{\operatorname{Im} U(s, b)}{|1-i U(s, b)|^{2}},
$$

i.e.

$$
\sigma_{\text {inel }}(s)=8 \pi \int_{0}^{\infty} b d b \frac{\operatorname{Im} U(s, b)}{|1-i U(s, b)|^{2}} .
$$

It should be noted that

$$
\operatorname{Im} U(s, b)=\sum_{n \geq 3} \bar{U}_{n}(s, b)
$$


where $\bar{U}_{n}(s, b)$ is a Fourier-Bessel transform of the function

$$
\begin{aligned}
\bar{U}_{n}(s, t)= & \frac{1}{n !} \int \prod_{i=1}^{n} \frac{d^{3} q_{i}}{q_{i 0}} \delta^{(4)}\left(\sum_{i=1}^{n} q_{i}-p_{a}-p_{b}\right) U_{n}^{*}\left(q_{1}, \ldots, q_{n} ; p_{a}^{\prime}, p_{b}^{\prime}\right) \cdot \\
& U_{n}\left(q_{1}, \ldots, q_{n} ; p_{a}, p_{b}\right) .
\end{aligned}
$$

Here the functions $U_{n}\left(q_{1}, \ldots, q_{n} ; p_{a}, p_{b}\right)$ and $U_{n}\left(q_{1}, \ldots, q_{n} ; p_{a^{\prime}}, p_{b^{\prime}}\right)$ correspond to the ununitarized (input or "Born") amplitudes of the process

$$
a+b \rightarrow 1+\ldots+n,
$$

and the process with the same final state and the initial state with different momenta $p_{a}^{\prime}$ and $p_{b}^{\prime}$

$$
a^{\prime}+b^{\prime} \rightarrow 1+\ldots .+n,
$$

respectively. They are the analogs of the elastic $U$-matrix for the processes $2 \rightarrow n$. The sum in the right hand side of the Eq. (8) runs over all inelastic final states $|n\rangle$ which include diffractive as well as non-diffractive ones. Graphically, these relations are illustrated in Fig. 1.
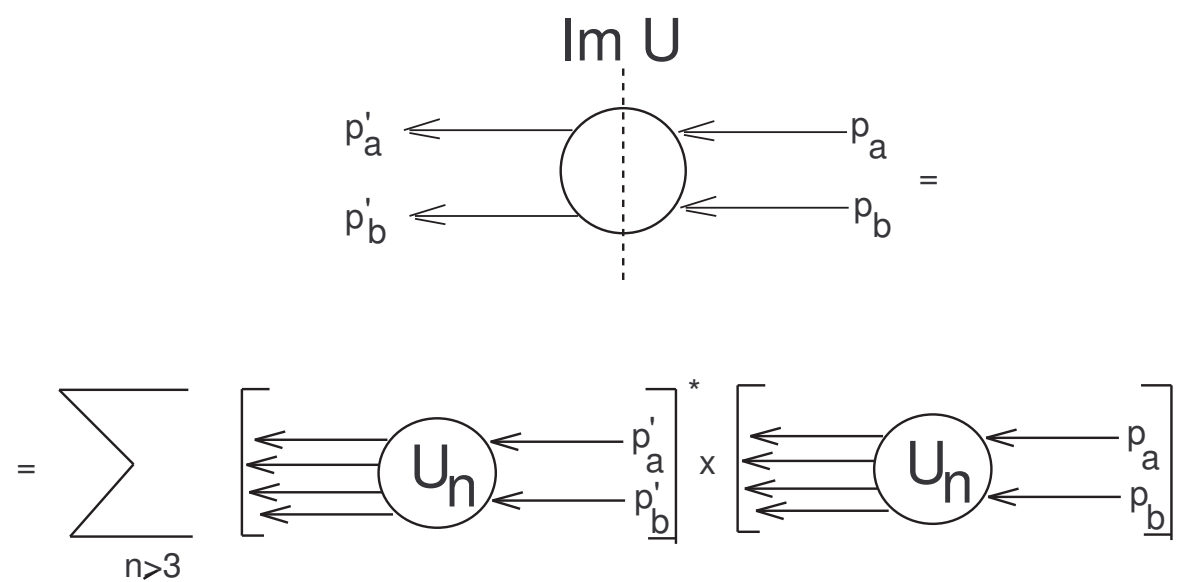

Figure 1: Unitarity for the function $\operatorname{Im} U$.

Then the inclusive cross-section of the process $a b \rightarrow c X$ has the following form [22]:

$$
E \frac{d \sigma}{d^{3} q}=8 \pi \int_{0}^{\infty} b d b \frac{I(s, b, q)}{|1-i U(s, b)|^{2}}
$$

where $I(s, b, q)$ is the Fourier-Bessel transform of the functions which are defined similar to Eq. (8) but with the fixed momentum $q$ and energy $E$ of the particle $c$ in the final state (Fig. 2). 


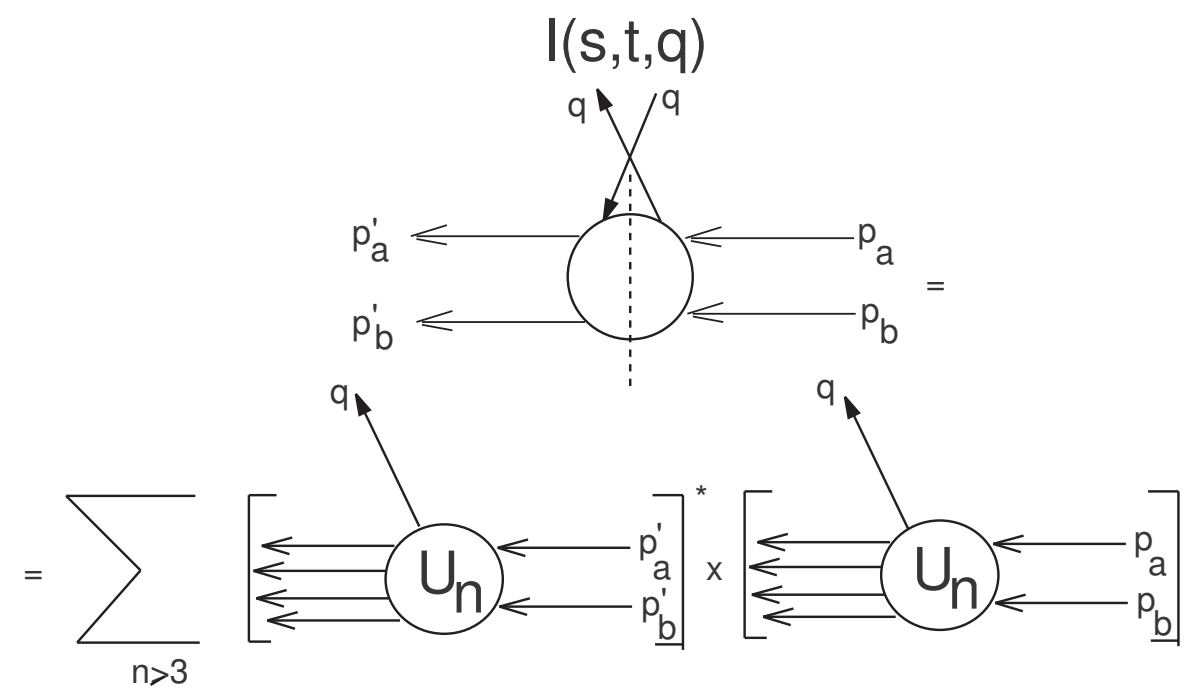

Figure 2: Unitarity for the function $I(s, t, q)$.

It should be noted that the impact parameter $b$ is the variable conjugated to the variable $\sqrt{-t}$, where $t=\left(p_{a}-p_{a}^{\prime}\right)^{2}$ and that the following sum rule is valid for the $I(s, b, q)$

$$
\int \frac{d^{3} q}{E} I(s, b, q)=\bar{n}(s, b) \operatorname{Im} U(s, b) .
$$

The impact parameter $b$ is related to the impact parameters of the secondary particles by relation [23]

$$
\mathbf{b}=\sum_{i=1}^{n} x_{i} \mathbf{b}_{i}
$$

where $x_{i}$ stands for the Feynman variable $x$ of the $i$-th particle. We consider the double-pomeron exchange processes of the following type

$$
p p \rightarrow p+X+p,
$$

where + denotes gap in rapidity and $X$ can be a particle or a system of particles. To obtain the cross-section of the DPE process (12) we should single out in the sum over all inelastic final states $|n\rangle$ the specific final states $|n\rangle_{d p e}$, which are the final states corresponding to the specific kinematics of the corresponding DPE process. Then denoting via $\zeta$ a set of kinematical variables which characterizes the kinematics of the final state in reaction (12), a similar to Eq. (9) expression can be written for the cross-section $d \sigma_{d p e} / d \zeta$

$$
\frac{d \sigma_{d p e}}{d \zeta}=8 \pi \int_{0}^{\infty} b d b \frac{I_{d p e}(s, b, \zeta)}{|1-i U(s, b)|^{2}} .
$$


The formula (13) is consistent with the experimental result of CDF on the independence of SP on the gap number. The function $U(s, b)$ is related to the elastic scattering amplitude and can, in principle, be extracted from the elastic scattering experimental data, however, in practice, the various models can be used to construct the function $U(s, b)$ and get its form at the energies where the data are absent at the moment. The models also needed to construct $I_{d p e}(s, b, \zeta)$. The ratio

$$
\int d \zeta I_{d p e}(s, b, \zeta) / \int \frac{d^{3} q}{E} I(s, b, q)=c(s, b)
$$

should be considered as a gap production probability. From the Eqs. (10) and (14) it follows that

$$
\int d \zeta I_{d p e}(s, b, \zeta)=c(s, b) \bar{n}(s, b) \operatorname{Im} U(s, b) .
$$

Unitarity of the scattering matrix implies, in principle, an existence at high enough energies of the new scattering mode - a reflective one. The physical interpretation of this scattering mode has been given recently in [15]. The rational form of unitarization [20] implies a smooth transition to the reflective scattering at small impact parameters at the LHC energies. To get quantitative results we use earlier constructed model for the $U$-matrix based on the ideas of chiral quark models [24]. The picture of a hadron consisting of constituent quarks in the central part of hadron embedded into quark condensate implies that overlapping and interaction of peripheral clouds occur at the first stage of hadron interaction. The impact parameter dependence of this interaction is determined by the function $D_{C}(b)$, which is a convolution of the two condensate distributions $D_{c}^{a}(b)$ and $D_{c}^{b}(b)$ inside the hadrons $a$ and $b$. We consider for simplicity the case of a pure imaginary amplitude, i.e. $U \rightarrow i u$. The function $u(s, b)$ is represented in the model as a product of the averaged quark amplitudes $\left\langle f_{Q}\right\rangle$,

$$
u(s, b)=\prod_{i=1}^{N}\left\langle f_{Q_{i}}(s, b)\right\rangle
$$

in accordance with the assumed quasi-independent nature of the valence quark scattering, $N$ is the total number of valence quarks in the colliding hadrons. The essential point here is the rise with energy of the number of the scatterers like $\sqrt{s}$. The $b$-dependence of the function $\left\langle f_{Q}\right\rangle$ has a simple form $\left\langle f_{Q}(b)\right\rangle \propto$ $\exp \left(-m_{Q} b / \xi\right)$. The generalized reaction matrix gets the following form

$$
u(s, b)=g\left(1+\alpha \frac{\sqrt{s}}{m_{Q}}\right)^{N} \exp \left(-\frac{M b}{\xi}\right),
$$


where $M=\sum_{Q=1}^{N} m_{Q}$. Here $m_{Q}$ is the mass of constituent quark, which is taken to be $0.35 \mathrm{GeV}$ 1. The model provides linear dependence on $\sqrt{s}$ for the total cross-sections, i.e. $\sigma_{t o t}=a+c \sqrt{s}$ in the limited energy range $\sqrt{s} \leq 0.5 \mathrm{TeV}$ and asymptotically, $\sigma_{\text {tot }} \propto \ln ^{2} s$, while $\sigma_{\text {inel }} \propto \ln s$ [25, 26].

Inelastic diffractive processes in the central region (DPE) occur in the peripheral region of the impact parameter space. Constituent quarks, which are responsible for elastic scattering, are supposed to be located in the central part. This fact and relations (14) and (15) allow one to assume factorization at the $U$-matrix level and write down the expression for $I_{d p e}(s, b, \zeta)$ in the form

$$
I_{d p e}(s, b, \zeta)=\sigma_{d p e}(s, b, \zeta) \operatorname{Im} U(s, b) \text {, }
$$

where $\sigma_{d p e}(s, b, \zeta)$ describes hard or soft "Born" cross-section of DPE processes. It should be stressed that the factorization (18) is assumed to be valid for both soft and hard DPE processes at the $U$-matrix level. We do not expect similar factorization, e.g. for DD processes. This factorization is different also from the factorization discussed in [19] at the $S$-matrix level which is considered to be valid for all diffractive processes with hard scale.

We consider hard DPE processes because they are the most interesting ones, i.e.

$$
\sigma_{d p e}(s, b, \zeta) \rightarrow \sigma_{H}(s, b, \zeta) .
$$

Thus, under construction of $\sigma_{H}(s, b, \zeta)$ we will suppose that the interaction occur at small distances. We can write down then the probability of hard interactions in the model as a convolution

$$
\sigma_{H}(s, b, \zeta)=\int D_{c}^{h_{1}}\left(\mathbf{b}_{1}\right) \sigma_{H}^{0}\left(s, \mathbf{b}+\mathbf{b}_{1}-\mathbf{b}_{2}, \zeta\right) D_{c}^{h_{2}}\left(\mathbf{b}_{2}\right) d \mathbf{b}_{1} d \mathbf{b}_{2},
$$

where $\sigma_{H}^{0}\left(s, \mathbf{b}+\mathbf{b}_{1}-\mathbf{b}_{2}, \zeta\right)$ is the cross-section of the hard condensate (parton) interactions. If the interaction is hard, than it is natural to choose

$$
\sigma_{H}^{0}\left(s, \mathbf{b}+\mathbf{b}_{1}-\mathbf{b}_{2}, \zeta\right) \simeq \sigma_{H}^{0}(s, \zeta) \delta^{2}\left(\mathbf{b}+\mathbf{b}_{1}-\mathbf{b}_{2}\right) .
$$

It means that hard interaction cross-section can be represented as a convolution integral

$$
\sigma_{H}(s, b, \zeta) \simeq \sigma_{H}^{0}(s, \zeta) \int D_{c}^{h_{1}}\left(\mathbf{b}-\mathbf{b}_{1}\right) D_{c}^{h_{2}}\left(\mathbf{b}_{1}\right) d \mathbf{b}_{1},
$$

which is similar to the model originally proposed by Chou and Yang [13]. This model can be considered, in fact, as a model based on hard parton interactions. It is useful to note here that the hardness of the inelastic interaction does not

\footnotetext{
${ }^{1}$ Other parameters have the following values: $g=0.24, \xi=2.5, \alpha=0.56 \cdot 10^{-4}$.
} 
imply the smallness of the impact parameter $\mathbf{b}$ (cf. Eq. (11)), the typical values of the impact parameter $b$ are determined by the condensate distributions $D_{c}^{h_{1}}$ and $D_{c}^{h_{2}}$ which are responsible for the central diffractive production and have mostly peripheral impact parameter dependence. The two-scale picture of hadron scattering proposed in [19, 27], where the core of hadron is responsible for hard interactions and peripheral part - for soft interaction, should definitely take place in elastic diffraction, while the hard interaction in the inelastic diffraction can take place in the peripheral region as well. Of course, the energy dependencies of soft and hard hadron interaction radii are different, the hard interaction radius of hadrons is energy independent, while the soft hadron interaction radius rises with energy as $\ln s$. It will lead therefore to the limit $R_{s}^{2}(s) \rightarrow 0$ at $s \rightarrow 0$.

After these qualitative remarks we would like to provide a quantitative estimates for the energy dependence of $R_{s}^{2}(s)$ for DPE processes. We adopt a most simple choice and assume that the condensate distributions $D_{c}^{h_{i}}$ over impact parameter $b$ is controlled by the pion mass, then the convolution

$$
D_{c}^{h_{1}} \otimes D_{c}^{h_{2}} \sim \exp \left(-m_{\pi} b\right) .
$$

In Eq. (20) we do not write down explicitly dependence on the parton momentum fraction $x$; one can suppose that the $x$ and $b$ dependencies are factorized (at the $U$-matrix level) and give contribution to the cross-section $\sigma_{H}^{0}(s, \zeta)$ which will be cancelled out in the dependence of $R_{s}^{2}(s)$. Now we calculate this dependence with the models for the function $U(s, b)$ and for the impact parameter dependence of the function $\sigma_{H}(s, b, \zeta)$. According to Eq. (21) we should calculate the ratio of the two integrals $\sigma_{H}(s, b, \zeta) \eta(s, b)$ and $\sigma_{H}(s, b, \zeta)$ over the variables $b$ and $\zeta$. The function $R_{s}^{2}(s)$ in the range from the Tevatron energies till the LHC energies and beyond this region is depicted on Fig. 3.

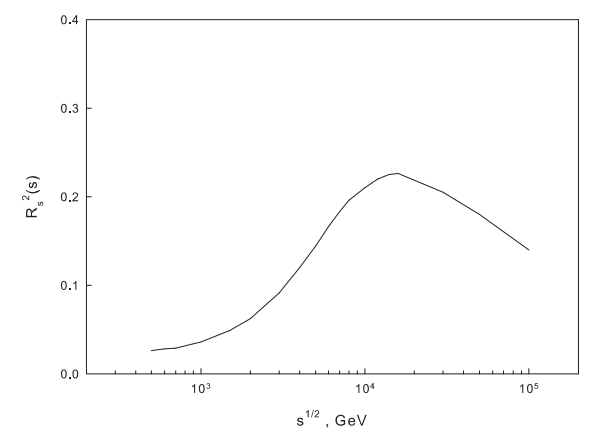

Figure 3: Energy dependence of survival probability $R_{s}^{2}(s)$.

Of course, numerical predictions for the gap survival probability obtained here are model dependent, but the qualitative picture of the energy dependence of $R_{s}^{2}(s)$ 
reflects transition to the new scattering mode at the LHC energies. It is interesting that situation is more favorable at the LHC energies since the obtained numerical values of $R_{s}^{2}(s)$ at these energies are close to maximal values around 0.2 and this should lead to higher cross-sections e.g. for Higgs production in DPE processes compared to the values obtained with eikonal estimations of the gap survival probability. Thus, appearance of the reflective scattering would result in better detecting of Higgs boson in DPE processes.

\section{Conclusion}

We would like to note that Eq. (2) is a more general definition of the gap survival probability than Eq. (1). Both are equivalent in the case of absorptive scattering, but they are different when the reflective scattering is present. The relevant definition in the latter case is Eq. (2).

The use of Eq. (2) leads to the value of $R_{s}^{2}(s) \sim 0.2$ for the hard DPE processes at the LHC energies instead of unity which has been obtained in [17] on the base of the definition Eq. (1). The energy dependence of $R_{s}^{2}(s)$ is determined by the interplay of soft and hard interaction radii. At $s \rightarrow \infty$ the ratio $R_{s}^{2}(s) \rightarrow 0$ due to dominance of the soft hadron interaction radius over the hard interaction radius of hadrons.

The LHC energies appear to lie in the favorable energy region, where soft and hard hadron interaction radii are close and this allows one to arrive to rather optimistic conclusions on the Higgs finding in the DPE processes there.

\section{Acknowledgement}

We are grateful to M. Arneodo, M. Grothe and V. Petrov for fruitful discussions on the subject of this note.

\section{References}

[1] Y. Dokshitzer, V. Khoze, S. Troyan, in Physics in Collision VI, Proceedings of the International Conference, Chicago, Illinois, 1986, edited by M. Derrick (World Scientific, Singapore, 1987), p. 365.

[2] A. Bialas, P.V. Landshoff, Phys. Lett. B256 (1991) 540.

[3] J. D. Bjorken, Phys. Rev. D45 (1992) 4077; Phys. Rev. D47 (1993) 101. 
[4] F. Abbot et al., (D0 Collaboration), Phys. Lett. B440 (1998) 189. M.E. Convery (for the CDF Collaboration), Acta Physica Polonica B, 36 (2005) 665;

K. Goulianos, FERMILAB-CONF-06-429-E.

[5] CMS Physics TDR, Volume II, CERN-LHCC-2006-021.

[6] V.A. Khoze, A.D. Martin, M.G. Ryskin, Eur. Phys. J. C26 (2002) 229.

[7] R. S. Fletcher, Phys. Lett. B320 (1994) 373.

[8] E. Gotsman, E. Levin, U. Maor, Phys. Lett. B438 (1998) 229.

[9] M.M. Block, F. Halzen, Phys. Rev. D63 (2001) 114004.

[10] A.B. Kaidalov, V.A. Khoze, A.D. Martin, M.G. Ryskin, Eur. Phys. J. C21 (2001) 521.

[11] V.A. Petrov, R.A. Ryutin, Eur. Phys. J. C36 (2004) 509.

[12] V.A. Khoze, A.D. Martin, M.G. Ryskin, hep-ph/0702213; A. De Roeck, V.A. Khoze, A.D. Martin, R. Orava, M.G. Ryskin, Eur. Phys. J. C25 (2002) 391.

[13] T.T. Chou, C.N. Yang, Phys. Rev. 170 (1968) 1591.

[14] J.S. Miller, hep-ph/0610427.

[15] S.M. Troshin, N.E. Tyurin, hep-ph/0701241.

[16] S.M. Troshin, N.E. Tyurin, Phys. Lett. B316 (1993) 175.

[17] S.M. Troshin, N.E. Tyurin, Eur. Phys. J. C39 (2005) 435.

[18] J. Bartels, S. Bondarenko, K. Kutak, L. Motyka, Phys. Rev. D73 (2006) 093004.

[19] L. Frankfurt, C.E. Hyde-Wright, M. Strikman, C. Weiss, Phys. Rev. D75, (2007) 054009.

[20] A.A. Logunov, V.I. Savrin, N.E. Tyurin and O.A. Khrustalev, Teor. Math. Phys. 6 (1971) 157.

[21] W. Heitler, Proc. Cambr. Phil. Soc., 37, 291 (1941).

[22] S.M. Troshin, N.E. Tyurin, Teor. Mat. Fiz. 28 (1976) 139 ; Z. Phys. C 45 (1989) 171.

[23] N. Sakai, Nuovo Cimento A 21 (1974) 368.

[24] S.M. Troshin and N.E. Tyurin, Nuovo Cim. 106A (1993) 327; Proc. of the Vth Blois Workshop on Elastic and Diffractive Scattering, Providence, Rhode Island, June 1993, p. 387; Phys. Rev. D49 (1994) 4427; Z. Phys. C 64 (1994) 311. 
[25] P.M. Nadolsky, S.M. Troshin, N.E. Tyurin, Z. Phys. C 69 (1995) 131.

[26] S.M. Troshin, N.E. Tyurin. Phys. Rev. D55 (1997) 7305.

[27] M.M. Islam, R.J. Luddy, A.V. Prokudin, Mod. Phys. Lett. A 18, (2003) 743. 